\title{
A Spectral-Scanning Nuclear Magnetic Resonance Imaging (MRI) Transceiver
}

\author{
Arjang Hassibi, Member, IEEE, Aydin Babakhani, Member, IEEE, and Ali Hajimiri, Senior Member, IEEE
}

\begin{abstract}
An integrated spectral-scanning nuclear magnetic resonance imaging (MRI) transceiver is implemented in a $0.12 \mu \mathrm{m}$ SiGe BiCMOS process. The MRI transmitter and receiver circuitry is designed specifically for small-scale surface MRI diagnostics applications where creating low (below $1 \mathrm{~T}$ ) and inhomogeneous magnetic field is more practical. The operation frequency for magnetic resonance detection and analysis is tunable from $1 \mathrm{kHz}$ to $37 \mathrm{MHz}$, corresponding to 0-0.9 $\mathrm{T}$ magnetization for ${ }^{1} \mathrm{H}$ (Hydrogen). The concurrent measurement bandwidth is approximately one frequency octave. The chip can also be used for conventional narrowband nuclear magnetic resonance (NMR) spectroscopy from $1 \mathrm{kHz}$ up to $250 \mathrm{MHz}$. This integrated transceiver consists of both the magnetic resonance transmitter which generates the required excitation pulses for the magnetic dipole excitation, and the receiver which recovers the responses of the dipoles.
\end{abstract}

Index Terms-Nuclear magnetic resonance (NMR) spectroscopy, magnetic resonance imaging (MRI), coherent detection, Torrey-Bloch equation, nuclear magnetic dephasing.

\section{INTRODUCTION}

$\mathbf{S}$ CALING down magnetic resonance imaging (MRI) [1] and nuclear magnetic resonance (NMR) spectroscopy systems [2] is a challenging task. Nonetheless, its realization has always been a goal in medical tomography imaging (3D imaging) due to its numerous potential applications in non-invasive imaging for the field of point-of-care $(\mathrm{PoC})$ diagnostics.

To design a portable magnetic resonance (MR) system, we are required to scale down all the components of the spectroscopy system, while keeping the sensitivity, detection time, spectral resolution, and the relative-to-sample-size spatial resolution (voxel resolution) the same compared to the large MR systems. This includes not only the necessary magnetization setup and the excitation and receiving coils, but also the electronic parts of the system which include the excitation pulse generators and the detection circuitry. The ability to operate in a non-uniform envelope magnetic field allows for elimination of the large and expensive superconductive magnets [3]-[5].

In order to explore the challenges of scaling down the MR systems, we need to first understand the scaling laws of MR signals. Through the reciprocity theorem in electromagnetic theory

Manuscript received March 18, 2008; revised February 23, 2009. Current version published May 28, 2009.

A. Hassibi is with the University of Texas at Austin, Austin, TX 78712-0323 USA (e-mail: arjang@mail.utexas.edu).

A. Babakhani and A. Hajimiri are with the California Institute of Technology, Pasadena, CA 91125 USA (e-mail: aydin@caltech.edu; hajimiri@caltech.edu).

Digital Object Identifier 10.1109/JSSC.2009.2020456

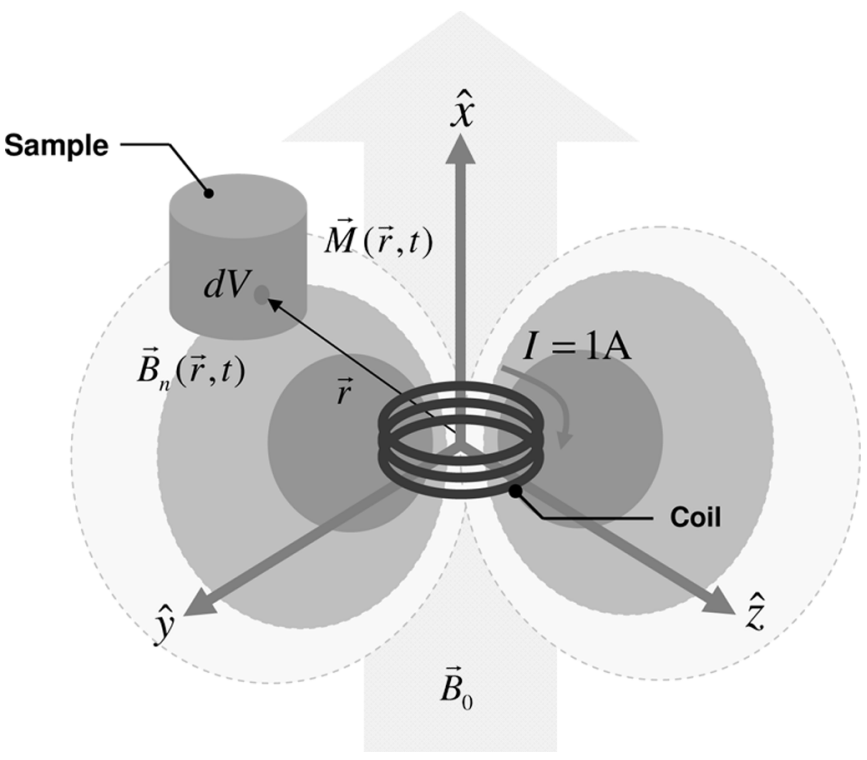

Fig. 1. Magnetic resonance spectroscopy system.

[1], the e.m.f. voltage induced in the coils of the MR system $E(t)$, can be described by the following:

$$
E(t)=-\int_{V} \frac{\partial}{\partial t}\left[\vec{B}_{n}(\vec{r}) \cdot \vec{M}(\vec{r}, t)\right] d V
$$

where $\vec{M}(\vec{r}, t)$ is the magnetic moment (and dipole) density created by the static magnetization field $\vec{B}_{0}$ in the sample volume $V$. The vector $\vec{B}_{n}(\vec{r})$ represents the magnetic coupling between the sample and the coils and is defined as the magnetic field generated by unit current in the coils at point $\vec{r}$ in the space (see Fig. 1). If we scale down this particular system in all dimensions by a factor $\alpha$, (i.e., scale down $V$ by the factor of $\alpha^{3}$ ), $\vec{M}(\vec{r}, t)$ stays unchanged since the density of the signal-generating magnetic dipoles (e.g., ${ }^{1} \mathrm{H}$ in proton MR spectroscopy) is independent of the volume. The effects of scaling on $\vec{B}_{n}(\vec{r})$ can be described using Ampere's Law, which indicates that $\vec{B}_{n}(\vec{r})$, to first order, is proportional to $\alpha^{-1}$. This means that by implementing scaled-down coils which are in intimate proximity of a scaled-down sample and without changing the magnetization strength we increase the $(\partial) /(\partial t)\left[\vec{B}_{n}(\vec{r}) \cdot \vec{M}(\vec{r}, t)\right]$ by $\alpha^{-1}$ while decreasing $E(t)$ by $\alpha^{2}$ due to volume reduction. This fundamental scaling law is very well understood in small volume NMR [3], [6]-[8] and high-resolution MRI systems [5], [9], [10].

Generally speaking, $\alpha$ has no direct relationship with the magnetizing field $\vec{B}_{0}$, since $\vec{B}_{0}$ is provided by a sample- and coil-independent source. From the Bloch equation [1], which 
describes the dynamics of the MR phenomenon and the relationship between $\vec{B}_{0}$ and $\vec{M}(\vec{r}, t)$, we know that $|\vec{M}(\vec{r}, t)| \propto\left|\vec{B}_{0}\right|$, i.e., the level of magnetization is proportional to the magnetization field. Clearly, by creating a larger $\vec{B}_{0}$, we can increase $\vec{M}(\vec{r}, t)$ and perhaps compensate for the $\alpha^{2}$ reduction in the resultant $E(t)$. This concept is currently being widely utilized in almost all high-resolution MRI systems. It is common to find huge magnetization setups, typically of the superconducting type, which are able to create magnetic fields in excess of $10 \mathrm{~T}$ up to $\vec{B}_{0}=11 \mathrm{~T}$. Unfortunately, this particular scheme to increase $E(t)$ and essentially signal-to-noise ratio (SNR) is somewhat impractical and costly for any small-scale and PoC application.

For portable NMR spectrometers and surface MR tomography systems, it is more practical to implement small rareearth magnets with up to $1.5 \mathrm{~T}$ peak magnetic field [10]-[14]. Even though these magnets can be manufactured in small customized shapes, the magnetic fields surrounding them are inherently inhomogeneous and non-uniform, which creates certain impediments for surface MR systems. The foremost is that when we place a sample containing certain magnetic dipoles (e.g., protons with resonance frequency of $42.58 \mathrm{MHz} / \mathrm{T}$ ) in such a field, we will observe a wide range of coordinate-dependant resonance frequencies. Clearly, such magnetization makes the conventionally "narrowband" magnetic resonance of that magnetic dipoles "wideband" depending on the range of variations in $\vec{B}_{0}$ through the sample. The second impediment is that the transversal relaxation of the dipoles becomes extremely short (i.e., fast decaying MR signal) in inhomogeneous magnetic fields. This is mainly due to the diffusion spreading of the dipoles, i.e., the magnetized dipoles can move to adjacent areas with slightly different resonance frequencies, resulting in de-phasing of the signal emanated from that particular volume. In Fig. 2, we show as an example the envelope decay of ${ }^{1} \mathrm{H}$ in water at room temperature as a function of magnetic field gradient, $G=\left|\nabla B_{0}\right|$ [8]. In this system, the zero-gradient $(G=0)$ relaxation time-constant $T_{2}^{*}$ is approximately $50 \mathrm{~ms}$, which is a typical value for ${ }^{1} \mathrm{H}$ in aqueous biological environments with $\left|\vec{B}_{0}\right|=1 \mathrm{~T}$. The additional envelope decay factor $f(t)$, which is due to $G$, is computed using the Torrey-Bloch formulations and is described by the following:

$$
f(t)=\exp \left(-\frac{1}{3} D \gamma^{2}|G|^{2} t^{3}\right)
$$

where $D=2.5 \times 10^{-9} \mathrm{~m}^{2}, \mathrm{~s}^{-1}$ is the diffusion coefficient of water molecules (and ${ }^{1} \mathrm{H}$ ) and $\gamma=2.6753 \times 10^{8} \mathrm{rad} \cdot \mathrm{T}^{-1} \mathrm{~s}^{-1}$ is the gyromagnetic ratio of ${ }^{1} \mathrm{H}$ in the water molecules [15]. For example, for typical rare-earth magnets used in surface MRI systems with dimensions of $2.5 \times 2.5 \times 2.5 \mathrm{~cm}^{3}$, the diverging magnetic field strength decays from $0.8 \mathrm{~T}$ to $0.4 \mathrm{~T}$ within a distance of $1 \mathrm{~cm}$ from the surface of the magnet, resulting in approximately $G=40 \mathrm{~T} \cdot \mathrm{m}^{-1}$, decay maximum rate of $0.1 \mathrm{~ms}$, and resonance frequency range of $34 \mathrm{MHz}$ to $17 \mathrm{MHz}$ for ${ }^{1} \mathrm{H}$. Clearly, if we are to perform any analysis of the MR response of a sample which is magnetized by this certain setup, we require a spectroscopy "transceiver" capable of operating within approximately one frequency octave and with a detection bandwidth of excess $10 \mathrm{kHz}$ to capture the signal before de-phasing.

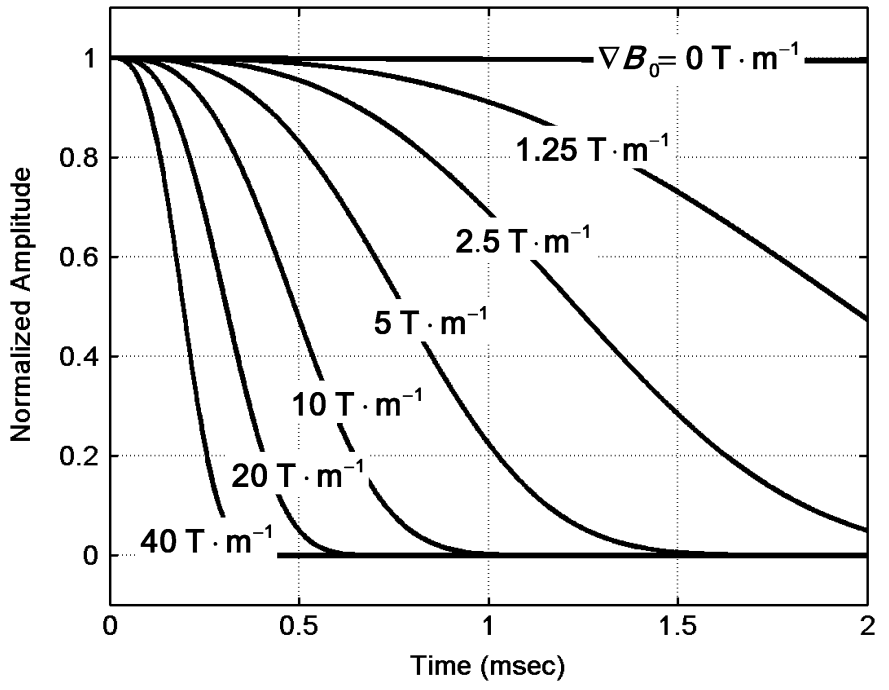

Fig. 2. Transversal relaxation (envelope decay) of the magnetic dipoles as a function of magnetic field gradient described by the Torrey-Bloch formulations.

It is important to recognize that the inhomogeneity of the magnetic field which results in the aforementioned impediments is somewhat irrelevant if the sample volume dimensions are much smaller than the rare-earth magnet dimensions, e.g., when we have sub-millimeter sample volume dimensions. Nevertheless, in the surface small-scale MRI systems, which we are focusing on in this paper, the sample volume size is comparable to the dimensions of the magnetization setup, and hence we need to take into account the problems associated to the magnetic field inhomogeneity.

Our goal in this paper is to show that we can leverage the capabilities of integrated circuits to address the practical challenges of small-scale MR systems, and to show the feasibility of designing an MR transceiver for $1-10 \mathrm{~cm}$ sample volume sizes. The basic idea is to design an integrated MR transceiver system-on-a-chip (SoC) that analyzes very wideband MR spectrums in real time and with high detection sensitivity. The wideband detection seems to be inevitable for small-scaled surface MRI platform using small, permanent and inevitably inhomogeneous magnetization setups. We also propose a "spectral-scanning" approach which exploits the non-uniformity of the magnetic field and extracts tomographic information from the resonance frequency of the dipoles [11], [16]. This is clearly in contrast with the single-tone and narrowband analysis approaches that conventional MRI and NMR systems utilize today.

To show the feasibility and proof of concept of such a system, we have designed, fabricated, and tested an integrated spectralscanning MR transceiver in a $0.12 \mu \mathrm{m} \mathrm{SiGe} \mathrm{BiCMOS} \mathrm{process.}$ The chip consists of two main blocks: (i) the MR transmitter which creates the wideband MR excitation signals, and (ii) the detector (or receiver) which detects the MR response signal of the sample. The excitation and detection coils are not integrated on-chip, since integrated coils (spiral micro-inductor structures) are not practical and area-efficient for our specified dimensions. Initially in Section II of this paper, we will introduce the proposed MR transceiver architecture in view of the system-level 
specifications of the spectral-scanning MRI (SSMRI) method. Subsequently, we will describe the designed circuit topologies, practical considerations, and measurement results in Section III.

\section{SySTEM-LEVEL DESIGN}

\section{A. Spectral-Scanning MRI (SSMRI) Method}

In the SSMRI method [16], the target object is placed within a deterministic inhomogeneous magnetic field created by a permanent magnet. Since the strength of the magnetic field $\vec{B}_{0}(x, y, z)$, is non-uniform within the imaging volume, the magnetic resonance frequency $\omega_{R}$ of identical magnetic dipoles (e.g., $\mathrm{H}^{1}$ or $\mathrm{C}^{12}$ ) within the target become coordinate-dependant, such that $\omega_{R} \propto\left|B_{0}(x, y, z)\right|$ for the volumetric pixel (voxel) at the coordinate $r=(x, y, z)$. Therefore, different coordinates within the target object can have dissimilar $\omega_{R}$ 's. We can extract certain tomographic information related to the target object by simultaneously measuring the resonance spectrum of particular nuclei within the imaging volume.

One important issue in the SSMRI method is that several voxels within the sample volume will share the same $\omega_{R}$, if they experience the same $\left|\vec{B}_{0}\right|$. Accordingly, in general there is not a one-to-one relationship between density of magnetic dipoles within the voxels and magnitude of the resonance frequencies in the measured spectrum. In general, all the points on an equi-magnetic field surface will have similar $\omega_{R}$ 's, making it necessary to obtain additional information to differentiate between the voxels on the same equi-magnetic field surface. This problem can be solved by changing the location of equi-magnetic field surfaces with respect to the sample and performing additional measurements. One can change $\vec{B}_{0}(x, y, z)$ within the object either by moving the relative position of the permanent magnet to the object, or introducing magnetic gradients similar to what conventional MRI generates.

To formulate this problem and construct an image using SSMRI, we consider an initial inhomogeneous magnetic field $\vec{B}_{0}(\vec{r})$, and given the excitation signals in time, the magnetic moment $\vec{M}(\vec{r}, t)$. If the normalized magnetic field of the $k$ th measurement at the voxel with coordinate $\vec{r}_{0}=\left(x_{0}, y_{0}, z_{0}\right)$ is $\vec{B}_{N}^{(k)}\left(\vec{r}_{0}\right)$, then the induced potential of this voxel at the receiving coil, $\varepsilon^{(k)}\left(t, \vec{r}_{0}\right)$, is

$$
\varepsilon^{(k)}\left(t, r_{0}\right)=-\frac{\partial}{\partial t}\left\{\vec{B}_{N}^{(k)}\left(\vec{r}_{0}\right) \cdot \vec{M}\left(\vec{r}_{0}\right)\right\} \Delta V
$$

where $\Delta V$ is the voxel volume. Clearly, $\varepsilon^{(k)}\left(t, \vec{r}_{0}\right)$ is relatively narrowband if $\Delta V$ dimensions are small compared to magnetic gradient in its volume and its center frequency is located around $\omega\left(\vec{r}_{0}\right)$, the resonance frequency at $\vec{r}_{0}$. For a gyromagnetic ratio of $\gamma$ this frequency is $\omega\left(\vec{r}_{0}\right)=\gamma\left|\vec{B}_{0}\left(\vec{r}_{0}\right)\right|$. It can be shown that magnitude of $\varepsilon^{(k)}\left(t, \vec{r}_{0}\right)$ is proportional to the magnetic of the dipole density at $r_{0}$, denoted by $\rho\left(\vec{r}_{0}\right)$, such that

$$
\varepsilon^{(k)}\left(t, r_{0}\right)=F^{(k)}\left(\vec{r}_{0}, t\right) \rho\left(\vec{r}_{0}\right) \Delta V
$$

where, based on $(3), F^{(k)}\left(\vec{r}_{0}, t\right)=-\partial\left[\vec{M}\left(\vec{r}_{0}\right) \cdot \vec{B}_{N}^{(k)}\left(\vec{r}_{0}\right)\right] / \partial t$. As is evident, this function is deterministic and is independent of $\rho$.
Now consider the imaging problem as finding $\rho$ within the imaging volume for $n$ finite voxels located at coordinates $\vec{r}_{0}, \vec{r}_{1}, \ldots, \vec{r}_{n-1}$ with volumes of $\Delta V_{0}, \Delta V_{1}, \ldots, \Delta V_{n-1}$. In addition, assume that we are observing the magnetic spectrum response of the system at $m$ different frequencies, $\omega_{0}, \omega_{1}, \ldots, \omega_{m-1}$, each having an induced signal $\mathrm{E}_{i}^{(k)}$ in the $k$ th experiment and at frequency $\omega_{i}$. We can define $\mathrm{E}_{i}^{(k)}$ as a function of $\rho$ using the following summation:

$$
\mathrm{E}_{i}^{(k)}=\sum_{j=1}^{n} f_{i, j}^{(k)} \rho_{j} .
$$

The function $f_{i, j}^{(k)}$ basically is very similar to $F^{(k)}\left(\vec{r}_{j}, t\right)$, yet it also includes the volume $\Delta V_{j}$ and the frequency of operation such that

$f_{i, j}^{(k)}= \begin{cases}F^{(k)}\left(\vec{r}_{j}, t\right) \Delta V_{j}, & \text { if } \omega_{i}-\delta \leq \gamma\left|B_{0}\left(\vec{r}_{j}\right)\right| \leq \omega_{i}+\delta \\ 0, & \text { else }\end{cases}$

where $2 \delta$ is the frequency bandwidth of $\varepsilon^{(k)}\left(t, \vec{r}_{j}\right)$.

It should be recognized that $f_{i, j}^{(k)}$, similar to $F^{(k)}(\vec{r}, t)$, is also a deterministic function and independent of function $\rho_{j}$. Now, by employing function $f_{i, j}^{(k)}$ as a scalar which relates $\rho_{j}$ to $\mathrm{E}_{i}^{(k)}$, we can define the following linear system:

$$
\begin{aligned}
\left(\begin{array}{c}
\mathrm{E}_{0}^{(k)} \\
\mathrm{E}_{1}^{(k)} \\
\mathrm{E}_{2}^{(k)} \\
\vdots \\
\mathrm{E}_{m-1}^{(k)}
\end{array}\right) & \left(\begin{array}{ccccc}
f_{0,0}^{(k)} & f_{0,1}^{(k)} & f_{0,2}^{(k)} & & f_{0, n-1}^{(k)} \\
f_{1,0}^{(k)} & f_{1,1}^{(k)} & f_{1,2}^{(k)} & \cdots & f_{1, n-1}^{(k)} \\
f_{2,0}^{(k)} & f_{2,1}^{(k)} & f_{2,2}^{(k)} & & f_{2, n-1}^{(k)} \\
& \vdots & & \ddots & \vdots \\
f_{m-1,0}^{(k)} & f_{m-1,1}^{(k)} & f_{m-1,2}^{(k)} & \cdots & f_{m-1, n-1}^{(k)}
\end{array}\right) \\
& \times\left(\begin{array}{c}
\rho_{0} \\
\rho_{1} \\
\rho_{2} \\
\vdots \\
\rho_{n-1}
\end{array}\right)
\end{aligned}
$$

or

$$
\mathrm{E}^{(k)}=F^{(k)} \cdot \rho
$$

where $\mathrm{E}^{(k)} \in R^{m}, F^{(k)} \in R^{m \times n}$, and $\rho \in R^{n}$.

To find $\rho$ we need to solve (7), given the measurement vector $\mathrm{E}^{(k)}$. Nevertheless, the criteria which makes this possible is $\operatorname{rank}\left(F^{(k)}\right) \geq n$. If this criteria is satisfied, we have enough (or even redundant) information to evaluate $\rho$. This is affected by the number of separate measurements to be performed, the magnetization setup, and how we alter $\vec{B}_{0}(\vec{r})$ within the imaging volume. Due to the larger number of independent measurements to be performed, it is more important for the implemented system to be able to generate and process information at multiple frequencies concurrently. In the next sections, we will focus on the transceiver design and implementation of SSMRI systems that meets such requirements.

\section{B. SSMRI Transceiver Specifications}

The basic specification for any MR systems is the frequency of operation (i.e., resonance frequency of the magnetic dipoles), which is essentially set by the strength of the magnetization field $B_{0}$ and the type of magnetic dipoles under analysis. If we are 


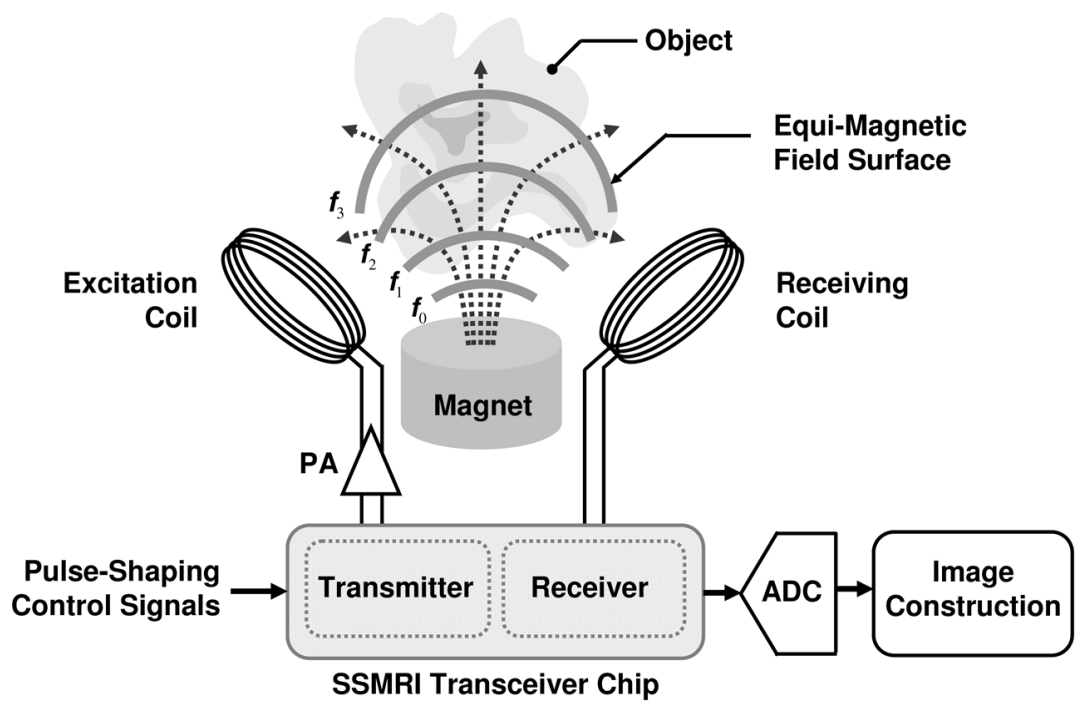

Fig. 3. The block diagram of the spectral-scanning MRI (SSMRI) system and SSMRI transceiver chip, where coordinate within equi-magnetic field surfaces translate to specific MR frequency channels.

to use permanent rare-earth magnets, the strength of the field is typically less than $1 \mathrm{~T}$ as mentioned before. The field gradients in this case, depending on how we assemble the magnetization setup, can vary from 0.01 to $40 \mathrm{~T} \cdot \mathrm{m}^{-1}$ in spatial coordinates where magnetic field strength has $10 \%$ to $80 \%$ of its maximum value. Such a range of magnetic field for proton $\left({ }^{1} \mathrm{H}\right) \mathrm{MR}$ spectroscopy translates to a frequency range of $4.3-34 \mathrm{MHz}$, which we set to be the operational frequency requirement for our SSMRI transceiver.

The bandwidth of MR detection, defined as the required bandwidth to properly acquire the transversal relaxation signal (envelope decay) of a certain dipole with a fixed $\vec{B}_{0}$, is a function of $T_{2}^{*}$ and $G$, as described in (1). For our system with $G=0.01-40 \mathrm{~T} \cdot \mathrm{m}^{-1}$, the maximum required bandwidth becomes $100 \mathrm{kHz}$, corresponding to the maximum decay of $10 \mu \mathrm{s}$, which we set as the target bandwidth for the MRI transceiver. This value also satisfies the requirements of $\delta$ and the voxel size described in (6).

One important issue regarding operating at the $4.3-34 \mathrm{MHz}$ frequency range is the high relative frequency of operation, which is greater than one octave and close to one decade. This potentially causes practical challenges in both the signal generation and detection circuitry of the SSMRI transceiver due to in-band high-order harmonics. Consequently, we have decided to keep the concurrent operation frequencies of the MR system within one octave, yet design the transceiver architecture such that it is capable of changing (sweeping) its center operation frequency to cover the whole 4.3-34 $\mathrm{MHz}$ frequency range.

In Fig. 3, we have illustrated the block diagram of the proposed spectral-scanning MRI transceiver. The transmitter in this system generates the wideband MR excitation pulses, and introduces them to the external excitation coil through an off-chip amplifier. The magnetization of the object under analysis, as discussed before, is carried out by implementing an inhomogeneous magnetic field with maximum magnetic field strength of $0.8 \mathrm{~T}$. In such a setup, the non-uniform magnetic field creates equi-magnetic surfaces with identical MR frequencies for a spe- cific magnetic dipole, and therefore forms a coordinate-to-frequency mapping for that magnetic dipole. The integrated receiver in the system detects the wideband MR signal (i.e., spectrum) that is induced in the receiving coil described by (1). As we discussed in the previous subsection, the detected MR spectrum of the object is subsequently used for image construction and evaluating tomographic information based on the resonance frequency and amplitude.

\section{IMPLEMENTATION}

\section{A. Transmitter}

Based on the specifications of our SSMRI transceiver, we are required to generate an excitation spectrum with a bandwidth just short of one octave, yet tunable and capable of covering the whole 4.2-34 MHz. In our design, we have implemented six tunable channels in the transmitter which collectively create the desired spectrum. In the transmitter (see Fig. 4) the excitation tone signals for all channels are generated by using a reference frequency and digital counters working as frequency dividers. The divisors for the digital counters ( 8 through 13 in our design) are selected such that the output frequencies of all 6 channels stay within one frequency octave to avoid complications related to the second-order harmonics. After tone generation using the digital dividers, we create the I and Q signals which are used in the transmitter and in the direct conversion receiver. The I and Q generator essentially is a divide-by-4 counter circuit, which results in overall frequency division ratios of $\{32,36,40,44,48$, $52\}$ in the channels. In Fig. 5, we have illustrated the frequency allocation of each channel within the output spectrum, and the step-by-step procedure in the transmitter to generate the excitation spectrum. In Fig. 6, we have illustrated the circuit architecture of a single channel in the transmitter. The reference frequency is recovered using a self-biased inverter which is shared among channels with measured lock-in sensitivity of approximately $-5 \mathrm{dBm}$ using a 64-pin leaded ceramic (LDCC) ceramic package, $3 \mathrm{~mm}$ bondwire and $50 \Omega$ source. 


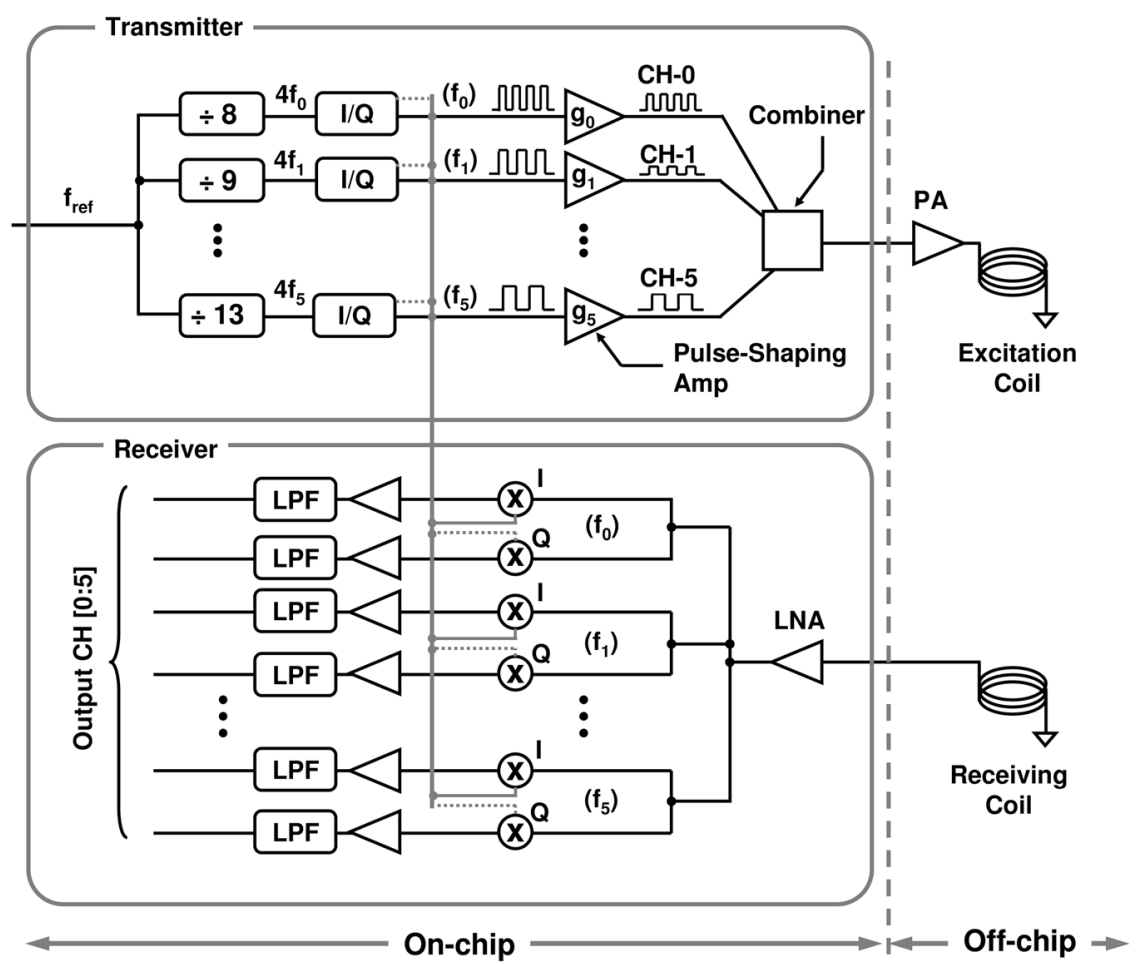

Fig. 4. Architecture of the spectral-scanning MRI transceiver.

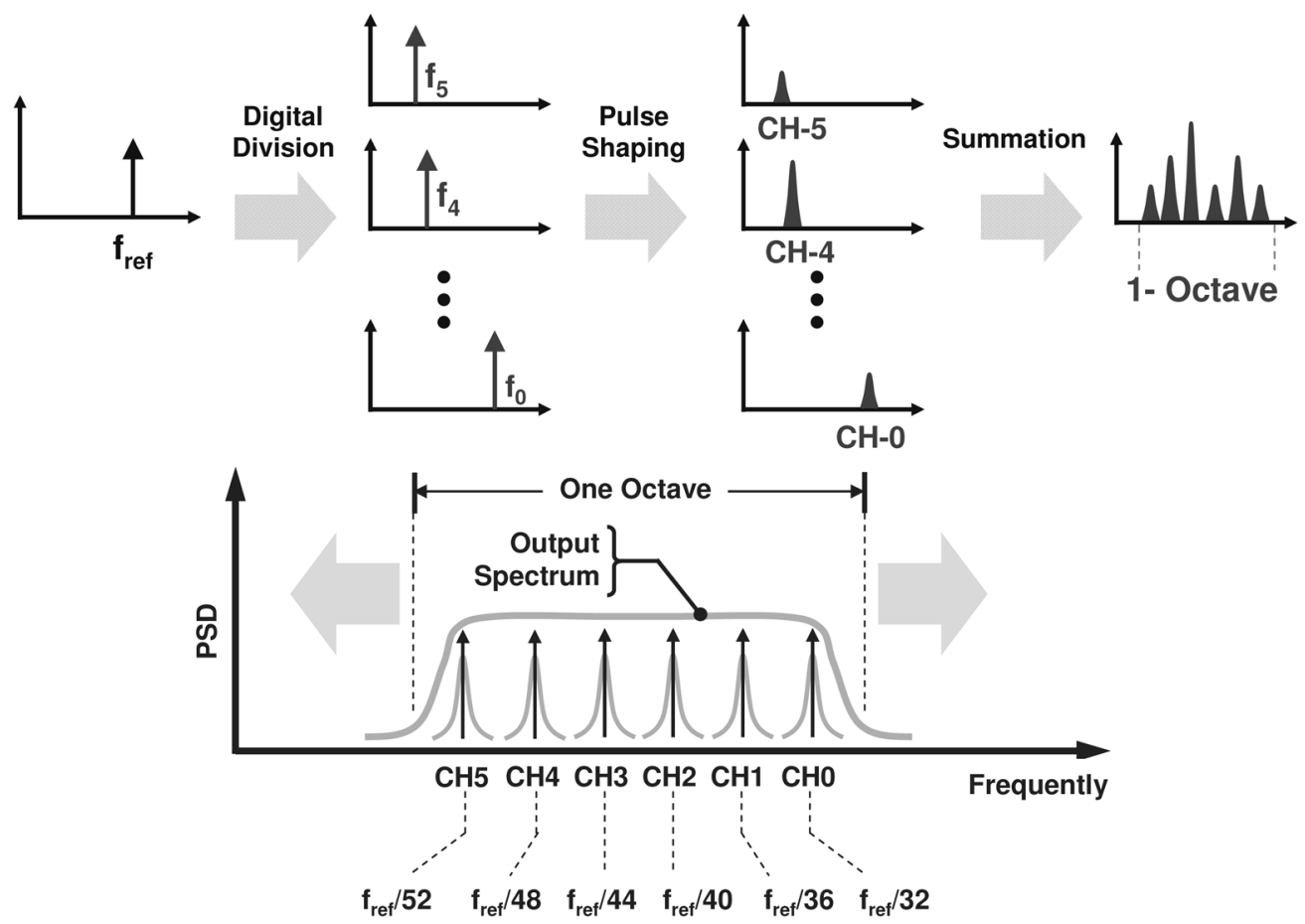

Fig. 5. Output spectrum profile of the transmitter.

Generally in MRI or NMR systems, the excitation sinusoidal pulses have a short duration with time-varying amplitude specifically designed based on the imaging technique and image reconstruction algorithm [1]. Accordingly, to satisfy this requirement in the transmit path of each channel we implement a synchronized enabling signal which activates the channel output with maximum switching frequency in excess of $50 \mathrm{MHz}$. The output amplitude can be further tuned to compensate for amplitude variations using a PMOS variable resistor as shown in Fig. 6. In Fig. 7, we have shown the output spectrum of Channel-0 (divisor equal to 32) of the transmitter when the $31.25 \mathrm{MHz}$ generated tone is switched on and off using the channel control signal with duration of $20 \mu \mathrm{s}$. The reference frequency in this case is $1 \mathrm{GHz}$. 


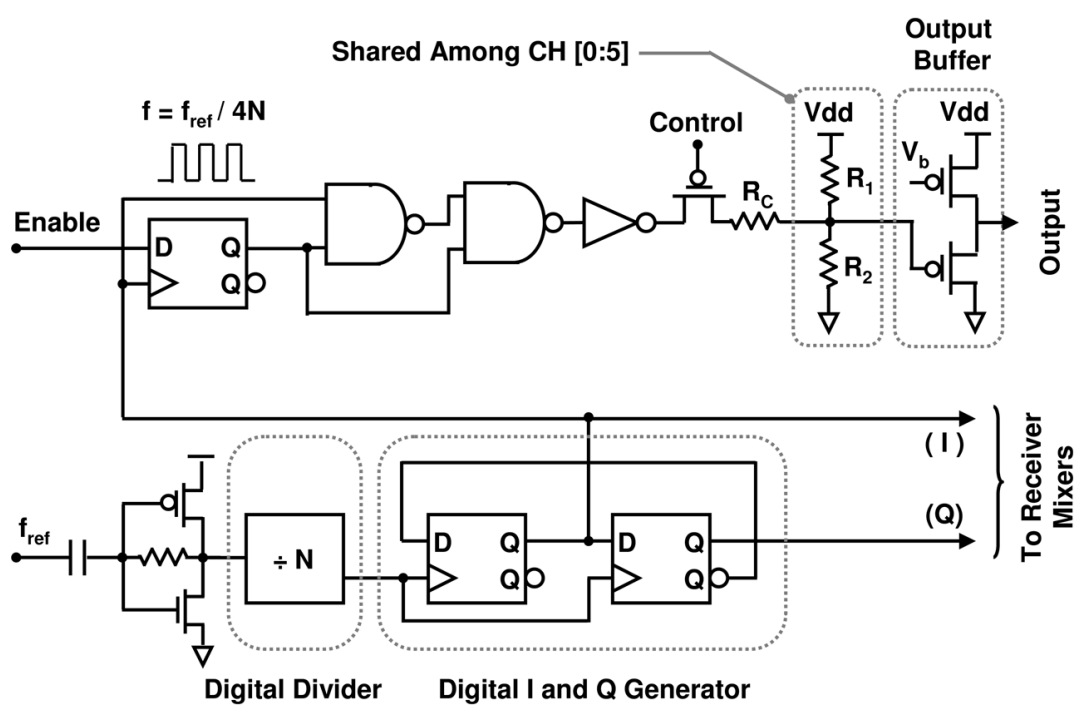

Fig. 6. Circuit architecture for a single channel of the transmitter.

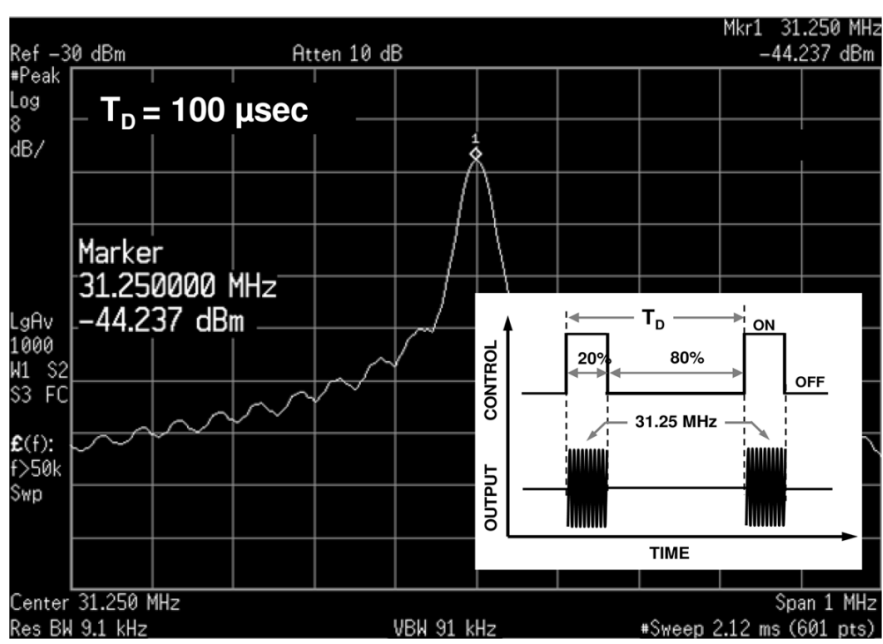

Fig. 7. Output spectrum and control signal of Channel-0 in the transmitter.

As shown in Fig. 6, in order to combine the outputs of all channels and create the excitation spectrum, we use a resistornetwork passive combiner followed by an output preamplifier. The maximum peak-to-peak voltage of the excitation signal for the combined channels at the combiner output with Vdd = $1.3 \mathrm{~V}$ is $3 \mathrm{mV}$, which is sufficient for driving the external RF amplifiers of the MR systems not integrated in this design. The measured output spectrum of the on-chip amplifier, when all six channels are operating without pulse shaping, is shown in Fig. 8 with, again, the reference frequency of $1 \mathrm{GHz}$.

The transmitter operation frequency range is from DC to $37 \mathrm{MHz}$ corresponding to an input reference frequency of DC to $1.2 \mathrm{GHz}$, respectively. The measured offset of the channels was approximately $2.3 \mathrm{~dB}$ which can be corrected through calibration. One important issue regarding SSMRI is the linearity of the transmitter. Basically the second-order harmonics and intermodulation (IM) products are out of band, since the excitation spectrum is limited within one frequency octave. However, the third-order intermodulation products will appear

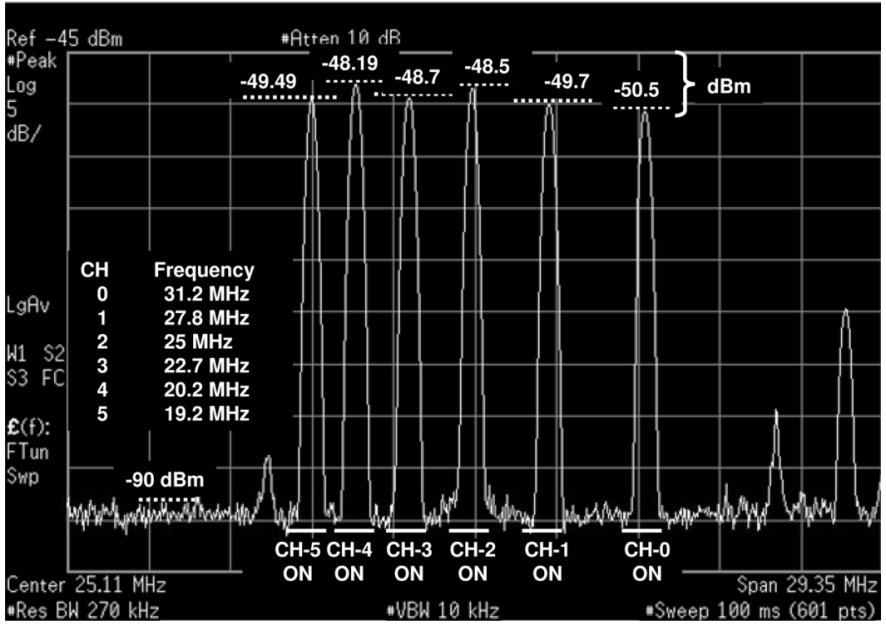

Fig. 8. Output spectrum and control signal of Channel-0 in the transmitter.

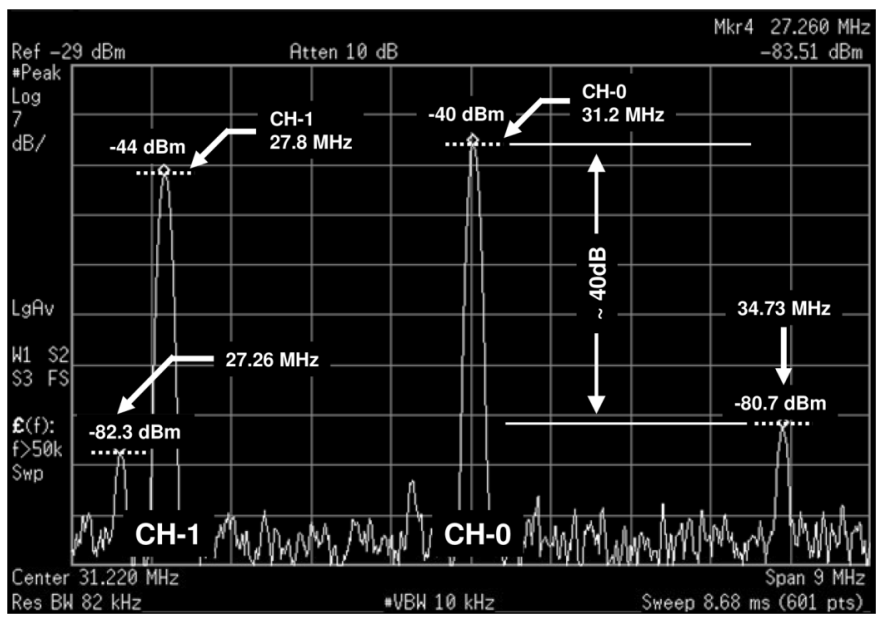

Fig. 9. The measured third-order intermodulation terms.

within the excitation spectrum. In our transmitter architecture, most of the nonlinear effects originate from the output buffer. In Fig. 9, we show the third-order IM products for channels 0 


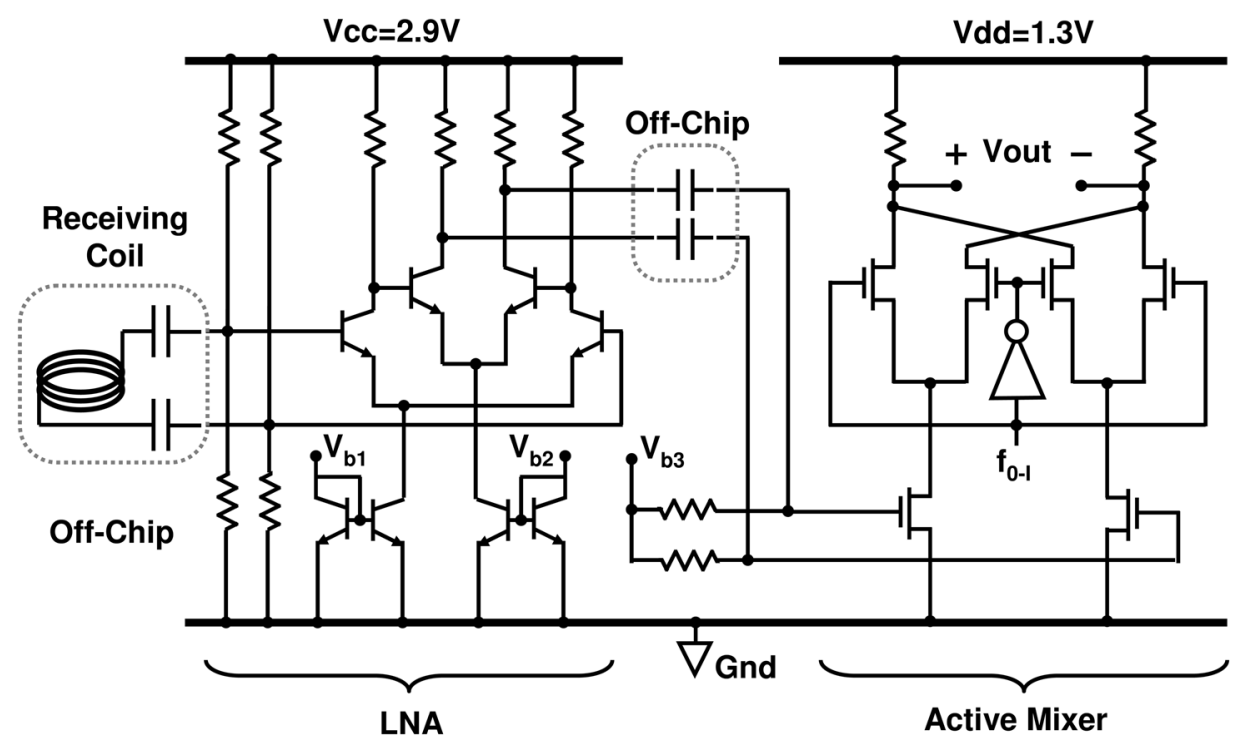

Fig. 10. The receiver circuit architecture for a single channel. The LNA is shared among all channels.

and 1 . The harmonics are less than $40 \mathrm{~dB}$ below channel levels which is sufficient for MR spectroscopy systems.

The overall power consumption of the transmitter block when all channels are active is a function of the reference frequency and monotonically increases from $6.5 \mathrm{~mW}$ at DC to $18.2 \mathrm{~mW}$ at $1.2 \mathrm{GHz}$ for $\mathrm{Vdd}=1.3 \mathrm{~V}$.

\section{B. Detector (Receiver)}

In the receiver, the signal induced into the receiving coils $E(t)$, which is the magnetic dipoles response to the excitation spectrum and $\vec{B}_{0}$, is initially amplified using a wideband LNA as illustrated in Fig. 10. To realize the LNA in our system, we have taken advantage of the bipolar NPN transistors of the SiGe BiCMOS process. The justification for this is the low noise characteristics, low 1/f noise corner, and the high gain of the NPN $\mathrm{SiGe}$ transistors. The LNA has a differential two-stage topology with resistive loads. The differential input of the LNA is biased internally, and there are two DC blocking external capacitors which connect the receiving coil to the input of the LNA. It is imperative to understand that the input impedance of the LNA in our design is not matched to the impedance of the coil (mostly inductive with less than $1 \Omega$ resistance) since the LNA is operating as a high input impedance amplifier, not a matched amplifier typically used in RF transceivers. The LNA measured gain is $31-27 \mathrm{~dB}$ at the frequency range of $10 \mathrm{MHz}$ to $300 \mathrm{MHz}$. The LNA has an input $1 \mathrm{~dB}$ compression point of $-21 \mathrm{dBV}$ with overall current consumption of $19.2 \mathrm{~mA}$ with $\mathrm{Vcc}=2.9 \mathrm{~V}$.

The measured input-referred voltage noise power spectral density (PSD) in the frequency range of $1 \mathrm{kHz}$ to $50 \mathrm{MHz}$ is $0.15 \mathrm{nV} / \sqrt{\mathrm{Hz}}$ and, based on simulations, remains constant up to $250 \mathrm{MHz}$. No flicker noise was observed above $1 \mathrm{kHz}$. The input-referred current noise PSD in the LNA has a magnitude of approximately $150 \mathrm{fA} / \sqrt{\mathrm{Hz}}$ based on simulations. It is important to recognize that the current noise in this particular design does not affect the noise performance of the LNA, since the impedance of the coil is significantly lower than LNA input

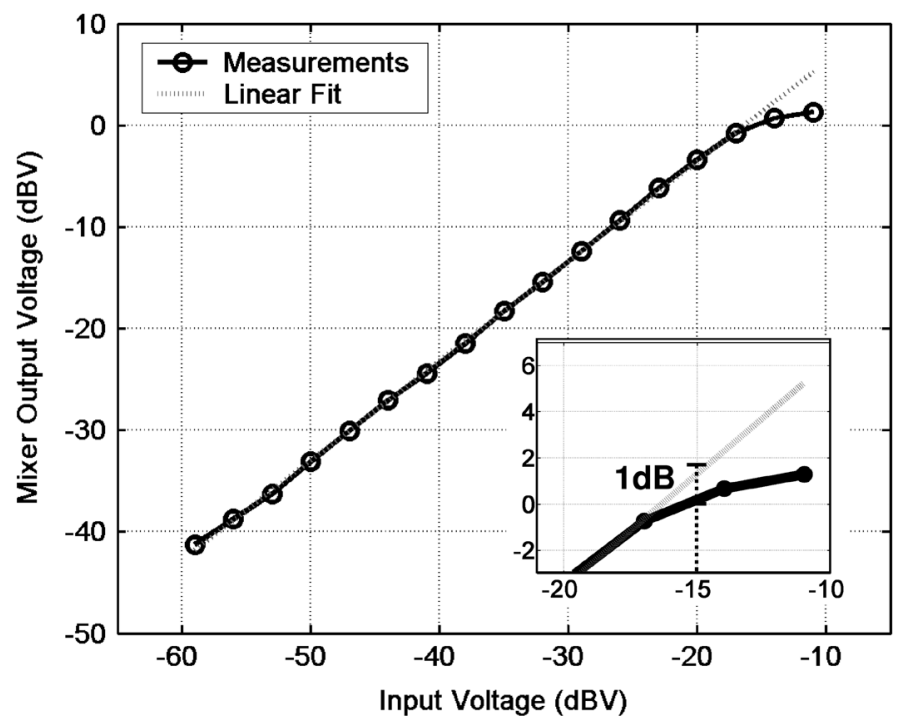

Fig. 11. Measured receiver gain and $1 \mathrm{~dB}$ compression point.

impedance and thus only the input-referred voltage noise gets amplified.

The output of the LNA, which amplifies the whole one octave spectrum, is connected to the mixers of all channels for down-conversion as shown in Fig. 3. The mixers in each channel have a CMOS active double-balanced topology with resistive loads (Fig. 10). The required LO frequency for the mixers comes from the transmitter block. In each receiving path of all six channels, there are two mixers which are driven by I and Q of that corresponding channel to create a coherent receiver. Using this method we are able to achieve $23 \mathrm{~dB}$ differential voltage gain for the mixers, with an input $1 \mathrm{~dB}$ compression point of $-15 \mathrm{dBV}$ for the whole receiver, limited by the mixer as shown in Fig. 11. The bandwidth of the mixer, which basically sets the bandwidth of the direct conversion receiver chain, is approximately $250 \mathrm{kHz}$ (see Fig. 12) which can be easily decreased by external capacitive loads to reach the $100 \mathrm{kHz}$ specification. 


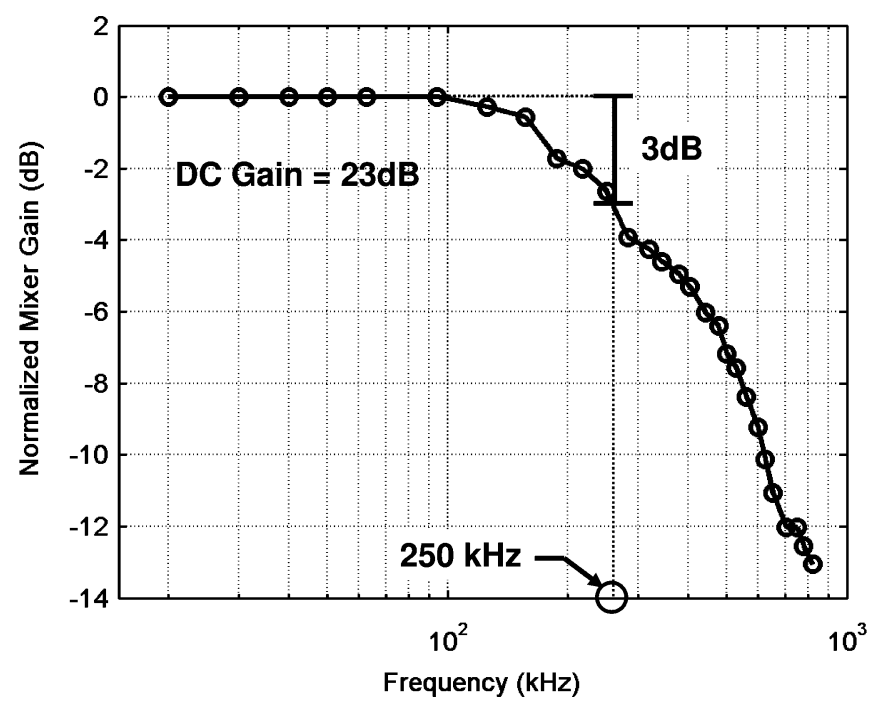

Fig. 12. Measured transceiver bandwidth for individual channel.

TABLE I

SPECTRAL-SCANNING MRI TRANSCEIVER SPECIFICATIONS

\begin{tabular}{|c|c|}
\hline System Specifications & \\
\hline Process & IBM $8 \mathrm{HP} 0.12 \mu \mathrm{m}$ SiGe BiCMOS \\
\hline Die specifications & $3 \mathrm{~mm} \times 3 \mathrm{~mm}, 88 \mathrm{pins}$ ( $56 \mathrm{I} / \mathrm{O}, 32$ test $)$ \\
\hline Off-chip & Coils, DC blocking capacitors, Output Power Amplifier \\
\hline Power consumption & $78.2-90 \mathrm{~mW}$ \\
\hline${ }^{1} \mathrm{H}$ detection & $1 \mathrm{kHz}-37 \mathrm{MHz}, B_{0}=0-0.85 \mathrm{~T}$ \\
\hline \multicolumn{2}{|l|}{ Transmitter } \\
\hline Operation frequency & MRI [1kHz-40MHz], NMR [1kHz-250MHz] \\
\hline Number of channels & MRI [0:5], Auxiliary [0:1] \\
\hline Output level & $0-3 \mathrm{mV} /$ channel, $I_{\mathrm{MAX}}=2.2 \mathrm{~mA}$ \\
\hline Modulation frequency & $50 \mathrm{MHz}$ \\
\hline In-band IM level & $-40 \mathrm{~dB}$ below channel \\
\hline Power consumption & $6.5-18.2 \mathrm{~mW}$ for $\mathrm{DC}-1.2 \mathrm{GHz}$ input frequency \\
\hline \multicolumn{2}{|l|}{ Receiver } \\
\hline Operation frequency & $<250 \mathrm{MHz}, \mathrm{BW}=250 \mathrm{kHz}$ \\
\hline LNA & Gain $=27 \mathrm{~dB}$, input noise $=0.15 \mathrm{nV} / \mathrm{Hz}^{1 / 2}$ \\
\hline Mixer & Gain $=23 \mathrm{~dB}, 1 \mathrm{~dB}$ comp $=-15 \mathrm{dBV}$ \\
\hline Power Consumption & $\mathrm{LNA}=55.5 \mathrm{~mW}$, Mixers $=16 \mathrm{~mW}$ \\
\hline
\end{tabular}

The overall gain of the receiver is $50 \mathrm{~dB}$ with $1 \mathrm{~dB}$ input compression point of $-42 \mathrm{dBV}$. The total power consumed in the receiver is $78.2-90 \mathrm{~mW}$ depending on the operation frequency from DC to $1.2 \mathrm{GHz}$. In Table I, we have listed all the measured specifications of the both the transmitter and the blocks which satisfies the requirement of the targeted spectral-scanning MRI systems.

In our system, for narrowband MR spectroscopy and electron spin resonance (ESR) spectroscopy [17] we have integrated two auxiliary channels which can function independently from the MRI transceiver. The major difference between these two channels and the spectral-scanning MRI transceiver is only in their transmitter. They do not have the initial digital dividers and their output is not combined with any other channel. Using these channels, narrowband signal excitation and coherent detection can be carried out up to $250 \mathrm{MHz}$ using the same LNA and mixers. The operation frequency in this mode is limited by the LNA bandwidth.

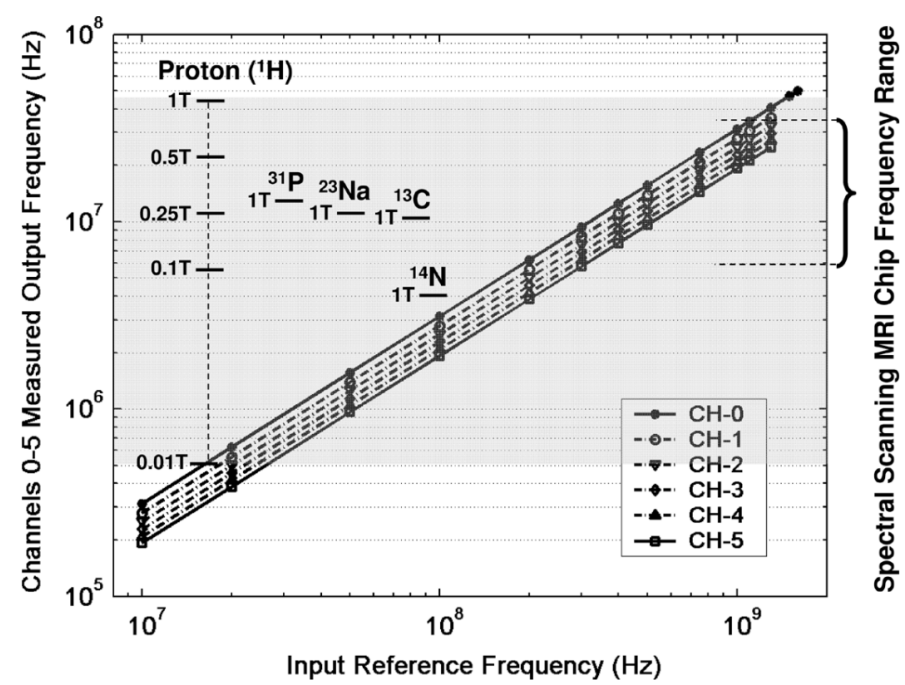

Fig. 13. The operation frequency of the spectral-scanning transceiver.

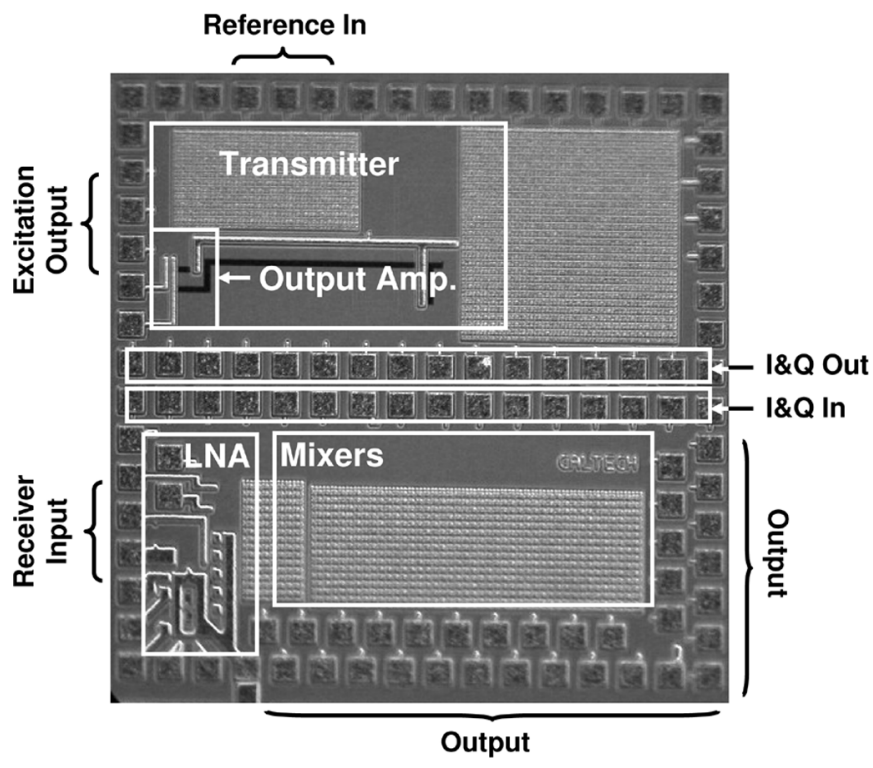

Fig. 14. Die micrograph of the SSMRI integrated transceiver chip.

In Fig. 13, we have illustrated the resonance frequency of various magnetic dipoles in different magnetic fields in addition to ${ }^{1} \mathrm{H}$ which is the most widely used in MRI systems. Clearly, the operational frequency of this transmitter is adequate for excitation of ${ }^{1} \mathrm{H}$ dipoles within $\vec{B}_{0}$ of less than $0.8 \mathrm{~T}$. However, other magnetic dipoles can also be excited (and detected) using this system since the spectroscopy method is identical and only the resonance frequency is different.

In Fig. 14, we show the die photo of our SSMRI integrated transceiver chip.

\section{CONCLUSION}

To scale down MRI and NMR systems into small-scale and desktop PoC systems, we face a number of fundamental challenges which originate not only from the MR scaling laws, but also the practical impediments of generating high magnetic fields in small volumes. In order to move toward realization of such a system and address these technical barriers, in this 
project, we have leveraged the capabilities of integrated systems to show the feasibility of an integrated spectral-scanning MRI (SSMRI) transceiver. The SSMRI method essentially exploits the inhomogeneity of the magnetic field to create a coordinate-to-frequency mapping and acquire tomographic information. The transceiver which we report in this paper is designed specifically for this method although it can be used for conventional NMR and MRI systems. The transceiver system not only has a very wide bandwidth of operation $(1 \mathrm{kHz}-37 \mathrm{MHz})$ but also can detect dipole relaxation decay time-constants as fast as to $0.4 \mu$ s with channel bandwidth of $250 \mathrm{kHz}$.

\section{ACKNOWLEDGMENT}

The authors would like to thank IBM Corporation for providing access to the $8 \mathrm{HP}$ BiCMOS process used in this project, and Hua Wang for technical feedback.

\section{REFERENCES}

[1] E. M. Haacke, R. W. Brown, M. R. Thompson, and R. Venkatesan, Magnetic Resonance Imaging: Physical Principles and Sequence Design. New York: Wiley, 1999.

[2] P. Callaghan, Principles of Nuclear Magnetic Resonance Microscopy. New York: Oxford, 1993.

[3] D. L. Olson et al., "High-resolution micro-coil ${ }^{1} \mathrm{H}-\mathrm{NMR}$ for masslimited, nanoliter-volume samples," Science, vol. 270, pp. 1967-1970, Dec. 1995.

[4] R. Magin et al., "Miniature magnetic resonance machines," IEEE Spectrum, vol. 34, no. 10, pp. 51-61, Oct. 1997.

[5] L. Ciobanu et al., "3D micron-scale MRI of biological cells," Solis Atate Nucl. Magn. Reson., vol. 25, pp. 138-141, Jan. 2004.

[6] G. Boero et al., "Fully integrated probe for proton nuclear magnetic resonance magnetometry," Rev. Sci. Inst., vol. 72, Jun. 2001.

[7] C. Massin et al., "High-Q factor RF planar microcoils for micro-scale NMR spectroscopy," Sensors Actuators A: Phys., vol. 97, pp. 280-288, Apr. 2002.

[8] J. Perlo et al., "High resolution NMR spectroscopy with a portable single-sided sensor," Science, pp. 1279-, May 2005.

[9] H. J. Mamin, M. Poggio, C. L. Degin, and D. Rugar, "Nuclear magnetic resonnace imaging with 90-nm resolution," Nature Nanotechnol., vol. 2, pp. 301-306, 2007.

[10] P. Prado, F. Casanova, and B. Blümich, "3D imaging with a singlesided sensor: An open tomograph,” J. Magn. Resonance, vol. 166, pp. 228-235, Feb. 2004

[11] A. Hassibi, A. Babakhani, and A. Hajimiri, "A spectral-scanning magnetic resonance imaging (MRI) integrated system," in Proc. IEEE Custom Integrated Circuits Conf. (CICC), 2007, pp. 123-126.

[12] L.-S. Fan et al., "Miniaturization of magnetic resonance microsystem components for 3D cell imaging," in IEEE ISSCC Dig., 2007, pp. 166-167.

[13] H. Lee, E. Sun, D. Ham, and R. Weissleder, "Chip-NMR biosensor for detection and molecular analysis of cells," Nature Medicine, vol. 14, no. 8, pp. 869-874, Aug. 2008.

[14] Y. Liu, N. Sun, H. Lee, R. Weissleder, and D. Ham, "CMOS mini nuclear magnetic resonance (NMR) system and its application for biomolecular sensing," in IEEE ISSCC Dig., 2008, pp. 140-141.

[15] V. M. Kenkre, E. Fukushima, and D. Sheltraw, "Simple solutions of the Torrey-Bloch equations in the NMR study of molecular diffusion," J. Magn. Resonance, vol. 128, pp. 62-69, Sept. 1997.

[16] A. Hajimiri, A. Hassibi, and H. Wang, "Spectral-scanning magnetic resonance imaging," US Patent App. No. 11/499, 921, Feb. 15, 2007.

[17] J. E. Wertz and J. R. Bolton, Electron Spin Resonance: Elementary Theory and Practical Applications. New York: McGraw-Hill, 1972.

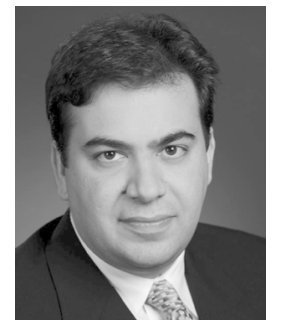

Arjang Hassibi (S'01-M'05) received the B.S degree with honors from the University of Tehran, Tehran, Iran, in 1997, and the M.S. and Ph.D. degrees from Stanford University, Stanford, CA, in 2001 and 2005 , respectively, all in electrical engineering.

From March 2005 to August 2006, he was a Postdoctoral Scholar with the Department of Electrical Engineering, California Institute of Technology. Since August 2006 he has been with the Department of Electrical and Computer Engineering of the University of Texas at Austin, where he is currently an Assistant Professor. He has also held research positions in Barcelona Design, Stanford Genome Technology Center, Panorama Research Institute, and Xagros Genomics which he co-founded in 2001. His main research areas are biosensors and bioelectronics, integrated sensors, DNA and protein integrated microarrays, biomedical VLSI systems, biological modeling, and noise spectroscopy.

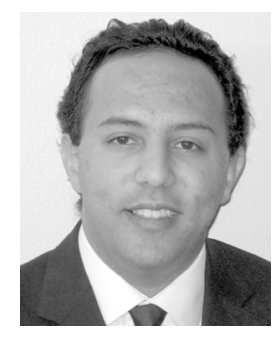

Aydin Babakhani (S'03-M'08) received the B.S. degree in electrical engineering from Sharif University of Technology, Tehran, Iran, in 2003, and the M.S. and Ph.D. degrees in electrical engineering from the California Institute of Technology (Caltech), Pasadena, CA, in 2005 and 2008, respectively.

Since 2008, he has been a Postdoctoral Scholar at Caltech working in the area of integrated circuits and antennas.

From 2006 to 2008, Dr. Babakhani was the Vice Chair of the IEEE Microwave Theory and Techniques Society Metro LA/SFV Joint Sections MTT-S Chapter 17.1. He was the recipient of the Microwave Graduate Fellowship in 2007, the Grand Prize in the Stanford-Berkeley-Caltech Innovators Challenge in 2006, ISSCC 2005 Analog Devices Inc. Outstanding Student Designer Award, as well as Caltech Special Institute Fellowship and Atwood Fellowship in 2003. He was also the Gold Medal winner of the National Physics Competition in 1998, as well as the Gold Medal winner of the 30th International Physics Olympiad in 1999 in Padova, Italy.

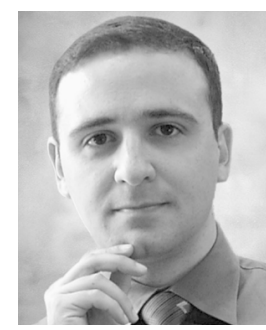

Ali Hajimiri (S'95-M'99-SM'09) received the B.S. degree in electronics engineering from the Sharif University of Technology, Tehran, Iran, and the M.S. and Ph.D. degrees in electrical engineering from Stanford University, Stanford, CA, in 1996 and 1998, respectively.

He was a Design Engineer with Philips Semiconductors, where he worked on a BiCMOS chipset for GSM and cellular units from 1993 to 1994. In 1995, he was with Sun Microsystems, where he worked on the UltraSPARC microprocessor's cache RAM design methodology. During the summer of 1997, he was with Lucent Technologies-Bell Labs, Murray Hill, NJ, where he investigated low-phase-noise integrated oscillators. In 1998, he joined the faculty of the California Institute of Technology, Pasadena, where he is a Professor of electrical engineering and the Director of Microelectronics Laboratory. He is a cofounder of Axiom Microdevices Inc. His research interests are high-speed and RF integrated circuits. He is the author of The Design of Low Noise Oscillators (Springer, 1999) and has authored or coauthored more than 100 refereed journal and conference technical articles. He holds more than 30 U.S. and European patents.

Dr. Hajimiri is a member of the Technical Program Committee of the IEEE International Solid-State Circuits Conference (ISSCC). He has also served as an Associate Editor of the IEEE Journal OF SOLID-STATE CIRCUITS, an Associate Editor of the IEEE TRANSACTIONS ON CIRCUITS AND SYSTEMS-PART II, a Guest Editor of the IEEE TRANSACTIONS ON MICROWAVE THEORY AND TECHNIQUES, a member of the Technical Program Committees of the International Conference on Computer Aided Design (ICCAD), and the Guest Editorial Board of Transactions of Institute of Electronics, Information and Communication Engineers of Japan (IEICE). 